\title{
Vitimas de bullying, sintomas depressivos, ansiedade, estresse e ideaçáo suicida em adolescentes
}

\author{
Fernanda de Oliveira Pimentel; Cristina Pilla Della Méa; Naiana Dapieve Patias
}

How to cite this article:

De Oliveira Pimentel, F., Della Méa, C.P., \& Dapieve Patias, N. (2020). Victims of bullying, symptoms of depression, anxiety and stress, and suicidal ideation in teenagers. Acta Colombiana de Psicología, 23(2), 205-216. http://www.doi. org/10.14718/ACP.2020.23.2.9

Recibido, marzo 01/2019; Concepto de evaluación, septiembre 16/2019; Aceptado, enero 16/2020

\author{
Fernanda de Oliveira Pimentel \\ IMED, Passo Fundo, Brasil \\ ORCID: https://orcid.org/0000-0003-2430-8408 \\ Cristina Pilla Della Méa* \\ IMED, Passo Fundo, Brasil \\ ORCID: https://orcid.org/0000-0002-8733-1560 \\ Naiana Dapieve Patias \\ Universidade Federal de Santa Maria, Santa Maria, Brasil \\ ORCID: https://orcid.org/0000-0001-9285-9602
}

Resumo

\begin{abstract}
Objetivou-se verificar a correlação entre ser vítima de bullying, ter sintomas depressivos, de ansiedade, de estresse e ideação suicida em adolescentes. Além disso, foi identificado se havia diferença nos construtos investigados, por gênero e idade. Estudo quantitativo transversal, do qual participaram 117 adolescentes. Utilizaram-se os instrumentos: questionário sociodemográfico, Escala Califórnia de Vitimização do Bullying, Escala de Depressão, Ansiedade e Estresse, e Escala Beck de Suicídio. As análises foram realizadas por meio de estatística descritiva, correlação de Spearman e teste U de MannWhitney. Os resultados indicam que 48,71 \% da amostra apresenta ideação suicida. Houve correlação positiva entre ser vítima de bullying e ter sintomas depressivos, ansiedade e estresse, e ser vítima e ter ideação suicida. As meninas apresentam mais sintomas de estresse do que os meninos, e adolescentes mais novos são mais vítimas de bullying do que os mais velhos. Destaca-se, assim, a relevância da discussão desse tema, tanto nas escolas quanto nas famílias, a fim de diminuir o sofrimento psíquico das vítimas.

Palavras-chave: bullying, adolescente, depressão, ansiedade, estresse, ideação suicida.
\end{abstract}

\section{Víctimas de bullying, sintomas depresivos, ansiedad, estrés e ideación suicida en adolescentes}

\begin{abstract}
Resumen
La presente investigación tuvo el objetivo de comprobar la correlación existente entre ser víctima de bullying y presentar síntomas de depresión, ansiedad, estrés e ideación suicida en adolescentes, así como identificar posibles diferencias para los constructos investigados según el género y la edad de los participantes. Para esto, se diseñó un estudio cuantitativo transversal, con una muestra de 117 adolescentes, donde se utilizaron instrumentos como la escala California de victimización de bullying, la escala de depresión, ansiedad y estrés, y escala de suicidio de Beck, así como un cuestionario sociodemográfico creado por las autoras. Los análisis descriptivos se realizaron a partir de medidas de estadística descriptiva, mientras que los
\end{abstract}

\footnotetext{
Rua Senador Pinheiro, 304, Passo Fundo, Rio Grande do Sul, Brasil. CEP: 990170-220. Telefone: (+55 54) 3054-6100. E-mail: cristina.mea@imed.edu.br
} 
correlacionales se hicieron con la prueba de Spearman y el test U de Mann-Whitney. Con esto, se encontró que el $48.71 \%$ de la muestra presenta ideación suicida; que hay una correlación positiva entre ser víctima de bullying y tener síntomas de depresión, ansiedad y estrés, así como entre ser víctima y tener ideación suicida; que las mujeres presentan más síntomas de estrés que los hombres; y que los adolescentes de menor edad son víctimas de bullying con mayor frecuencia. Finalmente, destaca la relevancia de la discusión sobre este tema tanto en las escuelas como en las familias, con el fin de disminuir el sufrimiento psíquico de las víctimas.

Palabras clave: bullying, adolescente, depresión, ansiedad, estrés, ideación suicida

\title{
Victims of bullying, symptoms of depression, anxiety and stress, and suicidal ideation in teenagers
}

\begin{abstract}
The study aimed to verify the correlation between being a victim of bullying, the presence of depression, anxiety, and stress symptoms and suicidal ideation in teenagers. Also, it intended to identify if there were differences between the variables investigated, by gender and age. This was a cross-sectional quantitative research in which 117 teenagers participated. The instruments used were: a sociodemographic questionnaire, the California Bullying Victimization Scale, the Depression, Anxiety and Stress Scale, and the Beck Scale for Suicidal Ideation. The analyzes were performed using descriptive statistics, Spearman correlation, and Mann-Whitney U test. The results indicate that $48.71 \%$ of the sample presented suicidal ideation. There was a positive correlation between being a victim of bullying and having depression, anxiety and stress symptoms, and being a victim and having suicidal ideation. Girls present more stress symptoms than boys and younger adolescents are more victims of bullying than older ones. Thus, discussing this issue is relevant, both in schools and in families, to reduce the psychological suffering of the victims.

Keywords: bullying, teenagers, depression, anxiety, stress, suicidal ideation.
\end{abstract}

\section{Introdução}

A adolescência é uma etapa do desenvolvimento na qual ocorrem mudanças biológicas, físicas e psicológicas. Além disso, por se tratar de um período de formação da identidade do jovem, é importante gerar um convívio social em que haja um ambiente seguro (Santrock, 2014). A relação entre os pares facilita o sentimento de pertencer a um grupo e promove o bom ajustamento biopsicossocial na vida adulta. Contudo, o estabelecimento de relações insatisfatórias contribui negativamente para as relações interpessoais futuras do jovem (Sprinthall \& Collins, 2008).

A violência e agressividade entre pares têm sido foco de atenção na atualidade, com repercussão na mídia, nas escolas e na sociedade como um todo. Esse fenômeno é definido como bullying e é manifestado de muitas formas (Calbo, Busnello, Rigoli, Shaefer, \& Kristensen, 2009; Silva \& Cabral, 2015). Normalmente, caracteriza-se por ações físicas e sociais intencionais e repetidas que são cometidas por uma ou mais pessoas contra um indivíduo que não consegue se defender facilmente(Dellazzana, Sattler, \& Freitas, 2010; Stelko-Pereira \& Williams, 2012; Waseenm \& Nickerson, 2017).

No Brasil, a Pesquisa Nacional de Saúde do Escolar de 2015 mostrou que a prevalência de vítimas de bullying na escola foi de 7,4\%, com chance maior de ocorrer em meninos, com idade de 13 anos, alunos da escola pública, adolescentes que relataram solidão (que não têm amigos), que apresentavam insônia, que sofreram agressões físicas de familiares, que tinham histórico de faltar aulas sem avisar os pais e que faziam uso de tabaco (Malta et al., 2019).

De maneira geral, o bullying no âmbito escolar pode envolver comportamentos físicos como bater, empurrar, socar, roubar lanche, usar armas para agredir; comportamentos verbais como ameaças, xingamentos e apelidos; comportamentos relacionais como a propagação de fofocas e mentiras que acabam por danificar o relacionamento com os pares. Também há o bullying indireto, o qual envolve comportamentos de exclusão, indiferença e extorsão. No que diz respeito aos papéis no cenário do bullying, estão: agressor, vítima, agressor-vítima e testemunha (Bandeira \& Hutz, 2012; Dellazzanna et al., 2010; Hui, Tsang, \& Law, 2011; Stelko-Pereira \& Williams, 2012). O agressor é a criança ou o adolescente que pratica o bullying em uma criança ou adolescente mais fraco (vítima), que, geralmente, não tem muitas condições físicas e emocionais para se defender. $\mathrm{O}$ (a) testemunha é quem que presencia as situações de bullying. Além dos papéis, há diferenças na forma como o bullying ocorre por gênero: o bullying físico 
é mais comum entre meninos, e o bullying relacional, em meninas (Bandeira \& Hutz, 2012).

Embora os estudos acerca do bullying sejam recentes, há indícios das consequências imediatas ou tardias do bullying na vida de crianças e adolescentes tanto na vitimização direta como na indireta (testemunhar). No que diz respeito à vida do adolescente em idade escolar, o bullying aparece como uma problemática comum, que abrange características associadas à área da saúde e ao cuidado no que tange às patologias que podem ser desencadeadas por esse motivo (Oliveira, Silva, Yoshinaga, \& Silva, 2015). Os danos emocionais à vítima podem ser a baixa autoestima e os sintomas depressivos se comparados aos de outros adolescentes que não passaram por tal intimidação (Sino et al., 2014). O grau de ansiedade também se mostra elevado (Malecki et al., 2015). Em casos mais graves, os efeitos são tão prejudiciais que resultam em tentativas de suicídio (Silva \& Cabral, 2015).

As consequências dessa vitimização podem ser observadas ainda no ensino fundamental, com a prevalência de sentimentos negativos, tais como: a vontade de não ir para a escola, o medo, a tristeza, a vontade de mudar de escola e a vontade de machucar o colega que o agrediu, o que pode vir a causar um impacto considerável no âmbito psicológico, físico, social e da aprendizagem (Santos, Perkoski, \& Kienen, 2015). Além disso, em muitos adolescentes, o bullying desencadeia transtornos depressivos, ansiedade e correlação com a maior probabilidade de desenvolver ideação suicida e, até mesmo, tentativas de suicídio (Moore et al., 2017). Transtornos depressivos, ansiedade e suicídio são efeitos do bullying que prevalecem com frequência na vítima até mesmo depois de este não ocorrer mais (Arseneault, 2017). Ainda, é visível a probabilidade da relação existente entre bullying e desenvolvimento dos sintomas do transtorno de estresse pós-traumático (Albuquerque, Albuquerque, Williams, \& D'Affonseca, 2013).

Considerado um problema de saúde pública, o bullying deve ser discutido em conjunto, pela escola e pela família dos adolescentes envolvidos, com ênfase na importância do respeito e da tolerância, para que se crie um ambiente de convívio saudável (Alves, 2016; Menegotto, Pasini, \& Levandowski, 2013; Silva \& Costa, 2016). É preciso, acima de tudo, conhecer o fenômeno do bullying e os efeitos deletérios dessa prática. Portanto, é dever de todos, principalmente dos pais (Hale, Fox, \& Murray, 2017) e das instituições de ensino, prezar pelo bem-estar das crianças e dos adolescentes (Segundo et al., 2016).

Assim, o presente estudo teve como objetivo investigar a correlação entre vitimização de bullying, sintomas depressivos, de ansiedade, de estresse e ideação suicida em adolescentes. Além disso, foi identificado se havia diferença, nos construtos investigados, por gênero e idade.

\section{Método}

\section{Tipo de estudo}

Estudo quantitativo, transversal, descritivo e correlacional (Breakwell, Hammond, Fife-Schaw, \& Smith, 2010).

\section{Participantes}

Por meio de amostragem não probabilística intencional, a partir da rede de contatos das pesquisadoras, participaram 117 adolescentes, com idades entre 13 e 17 anos $(M=15,14$ anos; $\mathrm{DP}=1,06)$ que estavam cursando o ensino fundamental ou o ensino médio em escolas de uma cidade localizada no norte do estado do Rio Grande do Sul, Brasil. Participaram da pesquisa apenas adolescentes que obtiveram autorização dos pais ou responsáveis por meio da assinatura do Termo de Consentimento Livre e Esclarecido (TCLE) e adolescentes que assinaram o Termo de Assentimento Livre e Esclarecido (Tale). Não participaram do estudo os adolescentes que não obtiveram autorização dos pais ou que estavam afastados das atividades escolares no período da coleta desta pesquisa.

Quanto aos dados sociodemográficos, a maioria dos adolescentes são do gênero feminino (71\%), de cor branca $(67,5 \%)$, solteiros $(99,1 \%)$ e moram com os pais $(72,6 \%)$. A família tem como renda o valor de $\mathrm{R} \$ 1.448,01$ a $\mathrm{R} \$ 2.172,00$ (26,5\%). A maior parte dos adolescentes estão cursando o $9^{\circ}$ ano (42\%) e estudam em escola pública $(64,1 \%)$, conforme a Tabela 1 .

No que diz respeito à realização de tratamento de saúde mental, $34 \%$ dos adolescentes já realizaram psicoterapia, e $7,7 \%$ dos adolescentes realizam tratamento psiquiátrico, dos quais $19 \%$ já fizeram uso de alguma medicação psiquiátrica, e $8 \%$ fazem o uso atualmente. Quanto ao psicofármaco utilizado, $6 \%$ fazem uso de antidepressivos, $3 \%$, de ansiolíticos e estabilizadores de humor, $2 \%$, de indutores do sono e $1 \%$, de psicoestimulantes. Em relação com os problemas de saúde, $17 \%$ dos adolescentes relataram ter algum problema de saúde: asma ou problema respiratório $(8 \%)$, renite alérgica $(2 \%)$ e problema de tireoide $(2 \%)$.

\section{Instrumentos}

Questionário Sociodemográfico. Construído pelas pesquisadoras a fim de mapear dados sociodemográficos como gênero, estado civil, idade, escolaridade, cor, com quem mora, se já realizou tratamento psicológico ou psiquiátrico, se tem algum problema de saúde, entre outros. 
Tabela 1.

Caracterização geral da amostra

\begin{tabular}{|c|c|c|c|}
\hline & Variável & $\mathrm{n}$ & $\%$ \\
\hline \multirow{3}{*}{ Gênero } & Feminino & 83 & 71 \\
\hline & Masculino & 34 & 29 \\
\hline & Branca & 79 & 67,5 \\
\hline \multirow[t]{2}{*}{ Cor } & Parda & 33 & 28,2 \\
\hline & Negra & 5 & 4,3 \\
\hline \multirow{2}{*}{ Estado civil } & Solttiro Casado/União estável & 116 & 99,1 \\
\hline & Solterro a asado/Uniao estaver & 1 & 0,9 \\
\hline \multirow{6}{*}{ Com quem mora } & Pais & 85 & 72,6 \\
\hline & Mãe & 12 & 10,3 \\
\hline & Pai & 2 & 1,7 \\
\hline & Avós & 3 & 2,6 \\
\hline & Mãe e padrasto & 13 & 11,1 \\
\hline & Outros & 2 & 1,7 \\
\hline \multirow{6}{*}{ Renda familiar } & Entre $\mathrm{R} \$ 0$ e $\mathrm{R} \$ 1.448,00$ & 22 & 188 \\
\hline & Entre $\mathrm{R} \$ 1.448,01$ e $\mathrm{R} \$ 2.172,00$ & 31 & 26,5 \\
\hline & Entre $\mathrm{R} \$ 2.172,01$ e $\mathrm{R} \$ 4.344,00$ & 28 & 24 \\
\hline & Entre $R \$ 4.344,01$ e $R \$ 7.240,00$ & 20 & 17 \\
\hline & Entre $R \$ 7.240,01$ e $R \$ 10.860,00$ & 12 & 10,3 \\
\hline & Acima de $\mathrm{R} \$ 10.860,00$ & 4 & 3,4 \\
\hline \multirow{4}{*}{ Escolaridade } & $9^{\circ}$ ano & 49 & 42 \\
\hline & $1^{\circ}$ ano do ensino médio & 21 & 18 \\
\hline & $2^{\circ}$ ano do ensino médio & 42 & 36 \\
\hline & $3^{\circ}$ ano do ensino médio & 5 & 4 \\
\hline \multirow{2}{*}{ Tipo de escola } & Particular & 42 & 35,9 \\
\hline & Pública & 75 & 64,1 \\
\hline
\end{tabular}

Fonte: elaboração própria.

Escala Califórnia de Vitimização do Bullying (ECVB). Instrumento adaptado por Soares, Gouveia, Gouveia, Fonsêca e Pimentel (2015), é composto por sete questões que indicam a frequência de comportamentos considerados bullying vivenciados no contexto escolar em uma escala Likert de 0 (nunca) a 4 (várias vezes). Em seguida, é avaliado se os comportamentos foram intencionais e capazes de magoar o(a) participante, com a utilização de respostas fechadas ("sim" e "não"). Ao final, a escala avalia o desequilíbrio de poder entre a vítima e o agressor por meio de dez adjetivos (exemplo: bonito, popular), e pede ao participante que se compare com a principal pessoa que realizou o comportamento de bullying, sendo as respostas: "menos que eu", "parecido comigo" e "mais do que eu". No estudo de validade de construto e consistência interna do instrumento, $o$ alfa de Cronbach da escola foi de $\alpha=0,72$. No mesmo estudo, a análise fatorial confirmatória corroborou estrutura unifatorial. No geral, o estudo de validade mostrou o grau de confiabilidade composta de 0,78 (Soares et al., 2015).

Escala de Depressão, Ansiedade e Estresse, versão adolescente (EDAE-A). Adaptada e validada para adolescentes brasileiros, a partir da versão Depression, Anxiety and Stress Scale - Short Form (DASS-21) utilizada para adultos brasileiros (Machado \& Bandeira, 2013). A escala possui 21 itens que avaliam sintomas de depressão, ansiedade e estresse. O participante indica o grau que experimentou o sintoma na última semana: de 0 ("não aconteceu comigo nesta semana") a 3 ("aconteceu comigo na maior parte do tempo nesta semana"). No estudo da adaptação da escala para adolescentes, foram testados três modelos presentes na literatura com o intuito de verificar a dimensionalidade da EDAE-A. O modelo que melhor se ajustou foi o original da escala, ou seja, o de três fatores, que representam as dimensões depressão, ansiedade e estresse 
$\left[\chi^{2}=366,16(186), p<0,001, \mathrm{CFI}=0,96, \mathrm{TLI}=0,96\right.$, RMSEA $=0,047(0,040-0,054)]$. A consistência interna apresentou, para cada fator, os seguintes alfas de Cronbach: 0,86 estresse, 0,83 ansiedade e 0,90 depressão (Patias, Machado, Bandeira, \& Dell'Aglio, 2016).

Escala de Ideação Suicida de Beck (BSI). Escala composta de 21 itens, pontuados de 0 a 3 , que detecta a presença de ideação suicida e mede a extensão da motivação e do planejamento de um comportamento suicida. Os dois últimos itens não são incluídos no escore final, mas fornecem informações sobre o indivíduo a respeito de tentativas de suicídio e da intenção de morrer. A escala não tem um ponto de corte; soma-se, então, a pontuação dos 19 itens; quanto mais altas as contagens, maior o risco de ideação suicida. Ao nível da consistência interna, os estudos psicométricos revelam um coeficiente alfa de Cronbach 0,96 (Cunha, 2001). A estimativa de fidedignidade da BSI, baseada no coeficiente alfa de Cronbach, numa amostra de adolescentes (não clínicos) para a BSI, foi de 0,94 (Werlang, Borges, \& Fensterseifer, 2004).

\section{Procedimento}

A coleta de dados se deu a partir da organização da agenda da coordenação de cada escola. No primeiro momento, a pesquisadora entrou nas turmas e fez o convite para os adolescentes participarem da pesquisa. Foram esclarecidos aos participantes a natureza e os propósitos do estudo, bem como a responsabilidade do pesquisador quanto ao sigilo da identidade do participante, podendo ser quebrado, conforme o código de ética profissional do psicólogo, e comunicado aos responsáveis ou à escola, para se promoverem medidas em benefício do indivíduo. Foram entregues e lidos com os adolescentes o TCLE e o Tale, de acordo com a Resolução 466/2012 e a 510/2016 do Conselho Nacional de Saúde do Brasil.

Posteriormente, foi combinada uma data para a aplicação dos instrumentos. A coleta ocorreu na sala de aula, de forma coletiva, somente com os alunos que trouxeram os termos assinados. Os participantes responderam ao questionário sociodemográfico e, em seguida, às demais escalas.

Após a coleta e análise dos dados, a pesquisadora que realizou a coleta fez a devolução dos dados aos participantes com a coordenação pedagógica e o psicólogo escolar (nas instituições que tinham esse profissional), a partir dos quais foram indicados os alunos que são vítimas de bullying e que apresentavam sintomas de depressão, de ansiedade, de estresse e ideação suicida. Na oportunidade, foi disponibilizado encaminhamento para a clínica-escola do curso de Psicologia da instituição do qual o estudo faz parte.

\section{Análise de dados}

Os dados foram analisados quantitativamente, com descrição e interpretação dos resultados pelo programa estatístico Statistical Package for the Social Sciences, versão 22.0. A análise descritiva e inferencial dos escores foi efetuada por meio das médias e dos desvios-padrão dos instrumentos, do teste U de Mann-Whitney, com o intuito de verificar diferenças, por gênero, nas variáveis investigadas, e da correlação de Spearman, para verificar a correlação entre idade e entre construtos investigados. A fim de verificar a normalidade das variáveis investigadas, foram considerados os valores de $p>0,05$ para o teste de Kolmogorov-Smirnov (Dancey \& Reidy, 2006). Como a distribuição das variáveis não atendeu aos pressupostos de normalidade, optou-se pela utilização de testes não paramétricos.

\section{Considerações éticas}

A pesquisadora obteve o aceite de quatro escolas (uma delas particular e as demais públicas) para realizar a pesquisa no referido local. Sobre os aspectos éticos, a pesquisa foi aprovada pelo Comitê de Ética e Pesquisa da Faculdade Meridional (Imed), com o Certificado de Apresentação para Apreciação Ética número 77923817.1.0000.5319.

\section{Resultados}

Quanto ao comportamento de vitimização de bullying ser intencional e ter magoado, a análise de frequência indicou que a maior parte dos adolescentes pontuou o quesito de ser deixado fora do grupo (41\%), seguido de rumores, boatos ou fofocas sobre você por seus colegas pelas suas costas (30 \%) e provocação ou apelido por parte de seus colegas (17\%), conforme a Tabela 2 .

Sobre as características dos colegas que realizaram os comportamentos descritos na Tabela 2 , no adjetivo "esperto", 31,6 \% responderam "parecido comigo"; no "fisicamente forte", $32,5 \%$ responderam "parecido comigo"; no "bonito", 30,8 \%, "parecido comigo" e, no "simpático", $39,3 \%$ pontuaram "parecido comigo". Ainda, houve a maior pontuação para "parecido comigo" no item "extrovertido" (38,5\%), "inteligente" (31,6\%) e "companheiro" (41\%). No instrumento, 32,5\% responderam "menos do que eu" na característica "atraente" e, na "magro", $34,2 \%$. Quando comparadas as afirmativas, por gênero, as meninas apresentaram maior escore nas afirmativas a seguir, que se caracterizam por bullying relacional: "Você teve rumores, boatos ou fofocas espalhados sobre você por seu(s) colega(s) pelas suas costas?" $(U=948,500$, $p<0,01)$; "Você foi deixado de fora do grupo ou ignorado 
Tabela 2.

Comportamento de vitimização de bullying intencional e que magoou a vítima

\begin{tabular}{lcc}
\hline Comportamento & $\mathrm{n}$ & $\%$ \\
\hline Provocação ou apelido por parte de seus colegas & 20 & 17 \\
Rumores, boatos ou fofocas sobre você por seus colegas pelas suas costas & 35 & 30 \\
Deixado de fora do grupo ou ignorado por seu(s) colega(s) & 48 & 41 \\
Empurrado ou agredido fisicamente & 3 & 3 \\
Ameaçado por seu(s) colega(s) & 0 & 0 \\
Teve suas coisas roubadas ou danificadas por seu(s) colega(s) & 6 & 5 \\
Comentários sexuais ou gestos correspondentes & 16 & 14 \\
\hline
\end{tabular}

Fonte: elaboração própria.

por seu(s) colega(s)?" $(U=1094,00 ; p=0,04)$ e "Você teve comentários sexuais ou gestos correspondentes dirigidos a você?" $(U=1137,00, p=0,03)$.

Quanto ao BDI, os resultados mostraram que 57 dos adolescentes (48,71\%) apresentam ideação suicida. A média foi de $1,75(D P=2,34)$, considerando-se que a presença é um escore diferente de zero, com variação do escore de 1 a 9 . Com o intuito de avaliar a gravidade na ideação suicida, foram somados os escores das afirmativas 1 a 19 . A variação nos escores variou de 3 a 28, sendo a média 12 $(D P=6,64)$. Quando comparada a gravidade na ideação suicida por gênero, meninas apresentaram maior gravidade do que os meninos $(U=1030,00, p=0,03)$.

Foram realizadas análises de correlação dos construtos investigados (sintomas de depressão, de ansiedade, de estresse, ideação suicida, vitimização do bullying) com a idade. O teste de Spearman indicou haver correlação apenas entre a idade e a vitimização do bullying, e foi considerada negativa e fraca, o que sugere que, com o aumento da idade, haja diminuição na vitimização ou quanto mais novo, maior a vitimização ( $r h o=-0,19 ; p=0,04)$. No entanto, essa correlação deve ser interpretada de maneira cautelosa pela magnitude da correlação encontrada e pelo tamanho amostral.

No que diz respeito ao gênero, o teste U de Mann-Whitney foi realizado com o intuito de verificar se havia diferenças, nos construtos investigados, entre meninos e meninas.
Apenas os sintomas de estresse apresentaram diferenças, com a apresentação de mais sintomas nas meninas do que nos meninos $(U=1035,500 ; p=0,02)$.

$\mathrm{O}$ teste de Spearman foi realizado com o intuito de verificar a correlação entre sintomas de depressão, de ansiedade e de estresse, vitimização do bullying e ideação suicida. A análise indicou haver correlação positiva, significativa e moderada entre ideação suicida e sintomas de depressão, de ansiedade e de estresse, e entre ideação suicida e vitimização do bullying, conforme a Tabela 3 .

\section{Discussão}

O presente estudo teve como objetivo investigar a correlação entre vitimização de bullying, sintomas depressivos, de ansiedade, de estresse e ideação suicida em adolescentes. Além disso, foi identificado se havia diferença, nos construtos investigados, por gênero e idade. Quanto à vitimização do bullying, a ECVB varia de 0 a 28 pontos; neste estudo, o escore foi de 0 a 16 pontos $(M=3,88 ; D P=3,76)$. Em estudo semelhante com este mesmo instrumento, no Mato Grosso, Brasil, 40,6 \% da amostra indicou ser vítima de bullying (Lima, 2013). Outra pesquisa que utilizou o Questionário de Exclusão Social e Violência Escolar, a média para a vitimização foi de 18,34 ( $D P=3,86)$ (Simões, Ferreira, Braga, \& Vicente, 2015).

Tabela 3.

Correlações de Spearmann entre sintomas de depressão, de ansiedade e de estresse, vitimização do bullying $e$ ideação suicida

\begin{tabular}{cccccc}
\hline & BSI total & ECVB total & Sintomas Depressão & Sintomas Ansiedade & Sintomas Estresse \\
\hline BSI total & - & $0,42^{* *}$ & $0,70^{* *}$ & $0,42^{* *}$ & $0,45^{* *}$ \\
ECVB total & $0,43^{* *}$ & - & $0,55^{* *}$ & $0,46^{* *}$ & $0,44^{* *}$ \\
\hline
\end{tabular}

Nota.** Todas as correlações da tabela apresentam valor de $p \leq 0,01$.

Fonte: elaboração própria. 
Em um estudo que avaliou prontuários de pacientes menores de 18 anos com queixas de saúde mental no departamento de emergência, em dois hospitais do Canadá, demostrou que $77 \%$ da amostra sofreu bullying em algum momento de suas vidas (Alavi et al., 2017). Em investigação semelhante, realizada em Nova York, Estados Unidos, participaram pacientes atendidos na emergência pediátrica com indicação de internação psiquiátrica, dos quais $13 \%$ relataram ser vítimas de bullying com frequência, definida como de duas a três vezes por mês ou mais (Leader, Singh, Ghaffar, \& Silva, 2018). Em outra pesquisa que envolveu adolescentes que frequentam a escola na Bolívia, na Costa Rica, em Honduras, no Peru e no Uruguai, a prevalência de qualquer vitimização nos últimos 30 dias foi de 37,8 \% (Romo \& Kelvin, 2016).

Ainda, referente à vitimização, uma pesquisa com adolescentes de 11 a 16 anos de um município do noroeste paulista, Brasil, com o questionário Kidscape, mostrou que quase metade da amostra (48,22 \% dos adolescentes) declarou ter sido alvo do bullying, a maioria do tipo verbal (46,56 \%) (Garbin, Gatto, \& Garbin 2016). Em estudo semelhante, realizado na Bahia, Brasil, apontou, por meio de autorrelato, que 76,75 \% dos alunos eram vítimas (Paixão et al., 2014). Em outra investigação, no Rio Grande do Sul, Brasil, 67,9 \% dos alunos declararam também serem vítimas de bullying (Fernandes \& Yunes, 2017). Em conjunto, os estudos indicam que muitos adolescentes estão sendo vítimas de bullying, dado preocupante, já que a vitimização tem consequências para o adolescente.

No presente estudo, em relação à idade, percebeu-se que, com o aumento dela, há a diminuição na vitimização, ou seja, quanto mais novo, maior a vitimização. Esse dado corrobora o estudo de Malta et al. (2014), que encontraram maior prevalência de bullying em estudantes com menos de 15 anos, acrescentando que, quanto mais jovens, maior a frequência desse comportamento (Malta et al., 2014). Outra pesquisa demostrou que alunos com 13 anos relataram terem sofrido mais bullying em comparação com outras faixas etárias, com redução após os 14 anos (Malta et al., 2019). A exposição dos adolescentes a atos de bullying é cada vez mais precoce, no entanto a tendência é diminuir conforme a idade aumenta (Moreno et al., 2012). Isso poderia ser justificado pelo fato de os alunos mais velhos conseguirem se defender sozinhos (Melim \& Pereira, 2013). Ainda, pode-se levantar a hipótese de que adolescentes mais novos relatem maior vitimização do que os mais velhos, já que a escala é de autorrelato.

Referente ao comportamento de vitimização de bullying intencional e que magoou a vítima, a maior parte dos participantes sinalizou o de "ser deixado fora do grupo" (41\%), seguido de "rumores, boatos ou fofocas sobre você por seus colegas pelas suas costas" (20\%). Os achados corroboram os resultados da pesquisa de Cavalcanti, Coutinho, Pinto, Silva e Do Bú (2018), que também utilizaram a ECVB e mostraram que o comportamento mais prevalente foi ter sido provocado ou ignorado $(60,9 \%)$ e ter sido vítima de rumores e piadas $(42,8 \%$ ), seguido por ter sido deixado de fora do grupo ou ignorado ( $42 \%$ ) em algum momento da vida escolar. No estudo de Leader et al. (2018), as vítimas referiram a exclusão e a disseminação de rumores como as formas mais frequentes de bullying.

A literatura mostra que a vítima de bullying aponta o fato de ser excluída por colegas e ser alvo de piadas e boatos maldosos como um dos maiores motivos de sofrimento psíquico (Agência Brasil, 2017). Nesse sentido, um estudo concluiu que os grupos que excluem essas vítimas são formados por colegas e amigos (33 \%) que acabam deixando-os de fora da prática de esportes ou de trabalhos escolares. As consequências em curto e longo prazo dessa exclusão para a vítima são limitação dos espaços que frequentam, dificuldade de socialização, baixo rendimento escolar e baixa autoestima (Santos \& Santos, 2013). É importante destacar que o grupo assume, nesse período da vida, um importante papel para adolescentes, já que faz parte do desenvolvimento, um distanciamento em relação aos pais e uma aproximação do grupo de iguais com o intuito de experimentações de outras regras e valores (DeSousa, Rodriguez, \& De Antoni, 2014).

Sobre os tipos de bullying, os estudos mostram que o verbal é mais comum do que o físico (Alavi et al., 2017; Bandeira \& Hutz, 2012), Garbin et al., 2016; Marcolino, Cavalcanti, Padilha, Miranda, \& Clementino, 2018; Vieira, Torales, Vargas, \& Oliveira, 2016). No presente estudo, 17\% dos adolescentes revelaram que se sentem magoados(as) pela "provocação ou apelido por parte de seus colegas". As agressões verbais são um tipo de violência mais velada, mais difícil de identificar e, consequentemente, de intervir; além disso, em muitos casos, é entendida como uma forma de brincadeira. Cabe ressaltar que ela não deve ser encarada como um comportamento de menor risco, ou seja, não deve ser minimizada, pois causa intenso sofrimento à vítima (Cavalcanti et al., 2018).

Neste estudo, os adolescentes vítimas de bullying indicaram que tinham características parecidas com os agressores, em que a resposta "parecido comigo" foi identificada em sua maioria, o que corrobora com a pesquisa de Bandeira e Hutz (2012), a qual mostrou que $54,7 \%$ dos alunos se identificaram com os agressores. Normalmente, a vitimização vem acompanhada de baixa autoestima pelo adolescente (Brito \& Oliveira, 2013), resultando em sentimentos de inferioridade quando se compara ao agressor (Santos \& Santos, 2013). A resposta "menos do que eu" foi pontuada 
212

somente na característica "magro" $(4,2 \%)$ e na característica "atraente" (32,5\%). Esse resultado pode ser justificado pelo fato de o instrumento ser de autorrelato, o que pode dificultar para o adolescente a expressão verdadeira do seu sentimento; além disso, pode-se questionar se a vítima não é, também, um agressor.

Referente ao bullying e ao gênero, os achados também foram confirmados por outros estudos, os quais demonstram que as agressões físicas são mais frequentes em meninos, enquanto nas meninas predominam formas mais sutis de agressividade, como as verbais, os insultos e as fofocas (Martins, 2009; Moreno et al., 2012). Outra pesquisa também denotou maior percentual de vitimização por bullying nos diversos tipos nas meninas (bullying verbal: $92 \%$ das meninas, $84 \%$ dos meninos; psicológico: $70 \%$ das meninas, $53 \%$ dos meninos; físico: $46 \%$ meninas, $44 \%$ meninos) (Brito \& Oliveira, 2013). Ainda, um estudo encontrou diferença significativa entre os sexos, o que mostra que os meninos utilizaram mais empurrões, chutes e socos, e as meninas utilizaram mais mentiras e fofocas (Bandeira e Hutz, 2012). Essa diferença de comportamento pode ser compreendida por questões culturais e pelas brincadeiras, já que as meninas ainda tendem a adquirir competências sociais de empatia e reconhecimento de vozes e rostos desde muito pequenas, e suas brincadeiras normalmente envolvem bonecas e de casinha, relacionando-se a interações sociais e verbais. Por sua vez, os meninos tendem a brincar mais com carrinhos, de bola e de luta, o que ressalta mais a questão física e mecânica, além de ser culturalmente aceito esse tipo de brincadeira, associado ao gênero (Seixas, 2009).

A diferença nos sintomas de estresse, por gênero, corrobora estudos que indicam que meninas apresentam mais sintomas de estresse do que meninos (Landstedt \& Gadin, 2012; Liu \& Lu, 2012; Machado, Veiga, \& Alves, 2011; Marques, Gasparotto, \& Coelho, 2015). Em uma investigação que relacionou estresse e tipos de envolvimento em violência escolar com 106 adolescentes de uma escola pública da cidade de Fortaleza, Brasil, também identificou maior média nas reações de estresse no gênero feminino quando comparadas ao masculino $(p<0,01)$ (Sousa \& Stelko-Pereira, 2016).

Um aspecto importante refere-se ao fato de que sintomas de estresse no ensino médio são mais prevalentes no gênero feminino e quanto à realização das atividades acadêmicas (Marques, Gasparotto, \& Coelho, 2015). Nessa mesma perspectiva, foram investigados sintomas de estresse em adolescentes com a Escala de Stress para Adolescentes, sendo a prevalência mais alta nas meninas em comparação com os meninos; além disso, destaca-se que a qualidade do relacionamento do adolescente com seus pais - em que estes valorizam e se interessam pelas atividades dos filhos-é um componente essencial para evitar esses sintomas na adolescência (Schermann et al., 2014).

Ainda, os resultados a respeito do gênero estão de acordo com o estudo de validação da escala para adolescentes brasileiros (Patias et al., 2016), no que diz respeito aos sintomas de estresse. No entanto, diferem dos resultados do estudo de validação quando são comparados com as diferenças encontradas nos sintomas de ansiedade e depressão. Esse aspecto pode indicar que os meninos, assim como as meninas, têm vivenciado sintomas que, anteriormente em pesquisas, eram comuns em meninas. No entanto, também pode ser levantada a hipótese de que não haver diferença, por gênero, nos sintomas de ansiedade e de depressão, no presente estudo, esteja relacionado ao pequeno tamanho amostral $(n=117)$ e à amostra ter mais meninas do que meninos (29\%).

Por sua vez, nesta pesquisa, os resultados do BSI mostram que $48,71 \%$ dos adolescentes apresentaram ideação suicida, com média de $1,75(D P=2,34)$. Os dados estão em consonância com a literatura, pois, em estudos semelhantes com a mesma escala, foi encontrada ideação suicida em 31,9 \% (Borges, Werlang, \& Copatti, 2008) e em $36 \%$ da amostra (Borges \& Werlang, 2006). Um estudo no Peru mostrou que $20 \%$ dos adolescentes apresentavam risco de suicídio, 15,2\% tinham histórico de tentativa de suicídio, 35,3\% tinham desejo passivo de morrer e 13,2\% tinham planejado suicídio em algum momento de suas vidas (Sandoval Ato, Vilela Estrada, Mejia, \& Caballero Alvarado, 2018). Contudo, em adolescentes avaliados em emergência pediátrica, há um aumento da prevalência de ideação suicida para 68,9 \% (Alavi et al., 2017).

É importante destacar que o suicídio é uma das maiores causas de morte na faixa etária entre 15 e 19 anos (Moreira $\&$ Bastos, 2015). O alto índice de suicídio na adolescência se justifica devido à pressão psicológica que os adolescentes sofrem nessa fase. Muitos deles veem o suicídio como uma única forma de alívio do sofrimento (Barbosa, Parente, Bezerra, \& Maranhão 2016).

Como fatores de risco para o suicídio na adolescência, destacam-se a solidão, a ausência dos pais, a influência dos amigos, o uso de álcool e de drogas (Ulbrich, Oselame, Oliveira, \& Neves, 2017). Ainda, conflitos familiares, problemas relacionados à escola, bullying, impulsividade e sintomas depressivos estão associados ao suicídio na adolescência (Sousa et al., 2017). No entanto, a correlação do bullying às ideias voltadas ao suicídio, principalmente pelas circunstâncias e pelas experiências que esse período do desenvolvimento proporciona, bem como pela intensidade com que vivem esses efeitos, pode levar a desfechos de autolesão. Assim, a prevenção quanto às diferentes formas 
em que se pode notar o bullying deve ser feita, para que esse índice diminua (Holt et. al., 2015).

No que diz respeito aos sintomas de depressão, de ansiedade e de estresse, tanto a vitimização quanto a ideação suicida se correlacionaram, de maneira moderada e positiva, com os sintomas de depressão, de ansiedade e de estresse, e entre ideação suicida e vitimização do bullying. Conforme Klomek et al. (2013), o bullying no ensino médio está entre os fatores de risco para os futuros problemas psiquiátricos e é forte tendência ao risco de depressão e do suicídio. De fato, uma pesquisa encontrou profunda correlação entre a vitimização e a internação psiquiátrica de adolescentes (Leader et al., 2018). Os resultados de Cavalcanti et al. (2018) confirmaram correlação positiva entre vitimização e sintomatologia depressiva $(p<0,01)$, e o estudo de Pabian e Vandebosch (2016) encontraram correlações positivas significativas com a vitimização e a ansiedade social $(p<0,001)$. Também Stapinski, Araya, Heron, Montgomery e Stallard (2015) associaram o fato de ser vítima com a elevação dos sintomas depressivos $(p<0,001)$ e de ansiedade $(p<0,001)$.

Além disso, os sintomas depressivos podem ser um dos reflexos da violência vivida pelos jovens e se manifestar por meio de nervosismo, pensamentos ruins, choro e ideação e tentativa de suicídio (Silva, 2010). Também os sintomas depressivos podem ser um indicativo de ser alvo ou autor do bullying (Forlim, Stelko-Pereira, \& Williams, 2014). Ainda, o bullying na adolescência pode desencadear transtorno depressivo, de ansiedade e a correlação a tentativas de suicídio ou a maior probabilidade de desenvolver ideação suicida (Moore et al., 2017). Os resultados desta pesquisa ainda indicam haver correlação positiva, significativa e moderada entre ideação suicida e sintomas, e entre ideação suicida e vitimização do bullying. Esses dados estão em consonância com demais pesquisas que demostram correlação significativa entre bullying e risco de suicídio $(p<0,001)$ (Sandoval Ato et al., 2018), entre vitimização e ideação suicida $(\operatorname{Pr}>\mathrm{Z}<0,0001)$ (Leader et al., 2018), entre bullying e suicídio $(p<0,01)$ (Alavi et al., 2017). Além disso, a vitimização foi associada a maiores probabilidades de ideação suicida com planejamento $(p<0,0001)$ e, pelo menos, uma tentativa de suicídio $(p<0,0001)$ (Romo \& Kelvin, 2016). Esses aspectos são importantes, pois indicam a necessidade de prevenção do bullying devido à sua magnitude e às consequências aos envolvidos.

O objetivo desta pesquisa foi investigar a correlação entre vitimização de bullying, sintomas depressivos, de ansiedade, de estresse e ideação suicida em adolescentes que frequentam a escola. $\mathrm{O}$ estudo indica dados preocupantes no que diz respeito à vitimização por bullying, à prevalência de sintomas de depressão, à ansiedade e ao estresse, principalmente em meninas, e à prevalência de ideação suicida na amostra. Esses dados contrariam a crença do senso comum e de muitos profissionais que ainda tendem a considerar o bullying uma "brincadeira".

Dessa forma, os resultados do estudo demonstram a importância de haver intervenção familiar e escolar não só para as vítimas de bullying, mas também para a comunidade escolar em geral, pois testemunhas e agressores também sofrem o fenômeno. No entanto, este estudo teve como foco a vitimização e indicou que ser vítima de bullying é algo sério e que não deve ter, de forma alguma, "passe livre" onde quer que seja, devido ao impacto significativo que traz para a vida desses jovens, como danos psicológicos, tanto emocionais como comportamentais. Além disso, embora não tenha sido o intuito deste estudo, foi possível revelar a ideação suicida de muitos adolescentes que foram encaminhados para serviços escolares. Por último, o não consentimento de todos os pais para a participação dos seus filhos na pesquisa, mesmo que estes quisessem fazer parte dela, foi um aspecto importante observado.

Como limitação deste estudo, pode-se destacar o fato de as escalas utilizadas serem de autorrelato, o que pode dificultar para o adolescente a expressão verdadeira do seu sentimento. Ainda, é importante que adolescentes agressores e espectadores possam fazer parte da pesquisa, já que neste estudo apenas a vitimização foi investigada.

Para futuros estudos, sugere-se a investigação de sintomas de depressão, de estresse, de ansiedade e ideação suicida nos adolescentes que praticam bullying e nos que o testemunham. A investigação sobre intervenções da escola diante dessas situações é outro aspecto relevante a ser investigado. Ainda, é importante que estudos futuros ampliem o tamanho amostral e tenham uma melhor distribuição, entre os gêneros, nos grupos.

\section{Referências}

Agência Brasil. (2017). É preciso falar sobre bullying, depressão e suicídio, alertam especialistas. http://agenciabrasil. ebc.com.br/geral/noticia/2017-04/e-preciso-falar-sobrebullying-depressao-e-suicidio-alertam-especialistas

Alavi, N., Reshetukha, T., Prost, E., Antoniak, K., Patel, C., Sajid, S., \& Groll, D. (2017). Relationship between bullying and suicidal behaviour in youth presenting to the Emergency Department. Journal of the Canadian Academy of Child and Adolescent Psychiatry, 26(2), 70-77. https:// www.ncbi.nlm.nih.gov/pmc/articles/PMC5510935/

Albuquerque, P. P., de Albuquerque, L. C., \& D'Affonseca, W. S. M. (2013). Efeitos tardios do bullying e Transtorno de Estresse Pós-Traumático: uma revisão crítica. 
Psicologia: Teoria e Pesquisa, 29(1), 91-98. doi:10.1590/ S0102-37722013000100011

Alves, M. G. (2016). Viver na escola: indisciplina, violência e bullying como desafio educacional. Cadernos de Pesquisa, 46(161), 594-613. doi:10.1590/198053143679

Arseneault, L. (2017). The long-term impact of bullying victimization on mental health. World psychiatry journal, 16(1), 27-28. doi: 10.1002/wps.20399

Bandeira, C. M., \& Hutz, C. S. (2012). Bullying: prevalência, implicações e diferenças entre os gêneros. Psicologia Escolar e Educacional, 16(1), 35-44. doi: 10.1590/ S1413-85572012000100004

Barbosa, A. K. L., Parente, T. D. L., Bezerra, M. M. M., \& Maranhão, T. L. G. (2016). Bullying e sua relação com o suicídio na adolescência. Journal and Psycology, 10(31), 1-19. doi: 10.14295/idonline.v10i31.501

Borges, V. R., \& Werlang, B. S. G. (2006). Estudo de ideação suicida em adolescentes de 15 a 19 anos. Estudos de Psicologia, 11(3), 345-351. doi: 10.1590/S1413-294X2006000300012

Borges, V. R., Werlang, B. S. G., \& Copatti, M. (2008). Ideação suicida em adolescentes de 13 a 17 anos. Barbarói, 28, 109123. doi:10.17058/barbaroi.v0i0.192

Breakwell, G. M., Hammond, S., Fife-Schaw, C., \& Smith, J. A. (2010). Métodos de pesquisa em Psicologia ( $3^{\mathrm{a}}$ ed.). Porto Alegre: Artmed.

Brito, C. C., \& Oliveira, M. T. (2013). Bullying and self-esteem in adolescents from public schools. Jornal de Pediatria, 89(6), 601-607. doi: 10.1016/j.jped.2013.04.001

Calbo, A. S., Busnello, F. de B., Rigoli, M. M., Schaefer, L. S., \& Kristensen, C. H. (2009). Bullying na escola: comportamento agressivo, vitimização e conduta pró-social entre pares. Contextos Clínicos, 2(2), 73-80. doi: 10.4013/ ctc. 2009.22 .01

Cavalcanti, J., Coutinho, M., Pinto, A., Silva, K., \& Do Bú, E. (2018). Vitimização e percepção do bullying: relação com a sintomatologia depressiva de adolescentes. Revista de Psicologia da IMED, 10(1), 140-159. doi:10.18256/21755027.2018.v10i1.2725

Cunha, J. A. (2001). Manual da versão em português das escalas Beck. São Paulo, SP: Casa do Psicólogo.

Dancey, C., \& Reidy, J. (2006). Estatística sem matemática para a psicologia: usando SPSS para Windows. Porto Alegre, RS: Artmed.

Dellazzana, L. L., Sattler, M. K., \& Freitas, L. B. (2010). A importância da inclusão da família na intervenção contra o bullying. Pensando Famílias, 14(1), 109-120.

DeSousa, D., Rodriguez, S., \& De Antoni, C. (2014). Relacionamentos de amizade, grupo de pares e tribos urbanas na adolescência. Em L. Habigzang, E. Diniz, \& S. Koller (eds.), Trabalhando com adolescentes: teoria e intervenção psicológica (pp.118-131). Porto Alegre, RS: Artmed.
Fernandes, G., \& Yunes, M. Â. M. (2017). O bullying no ambiente escolar: uma realidade a ser enfrentada. Resumos $d a$ Semana Cientifica Unilasalle. Canoas, RS: Unilasalle.

Forlim, B. G., Stelko-Pereira, A. C., \& Williams, L. C. de A. (2014). Relação entre bullying e sintomas depressivos em estudantes do ensino fundamental. Estudos de Psicologia (Campinas), 31(3), 367-375. doi:10.1590/0103-166x2014000300005

Garbin, C. A. S., Gatto, R. C. J., \& Garbin, A. J. I. (2016). Prevalência de bullying em uma amostra representativa de adolescentes brasileiros. Archives of Health Investigation, 5(5), 256-261. doi: 10.21270/archi.v5i5.1701

Hui, E. K. P, Tsang, S. K. M, \& Law, B. C. M. (2011). Combating school bullying through developmental guidance for positive youth development and promoting harmonious school culture. Scientific World Journal, 11, 2266-2277. doi: 10.1100/2011/705824

Hale, R., Fox, C. L., \& Murray, M. (2017). As a parent you become a tiger: parents talking about bullying at school. Journal of Child and Family Studies, 26(7), 2000-2015. doi:10.1007/s10826-017-0710-z

Holt, M. K.,Vivolo-Kantor, A. M., Polanin, J. R., Holland, K. M., DeGue, S., Matjasko, J. L., ... Reid, G. (2015). Bullying and suicidal ideation and behaviors: a metaanalysis. Pediatrics, 135(2), 496-509. doi: 10.1542/ peds.2014-1864

Klomek, A.B., Kleinman, M., Altschuler, E., Marrocco, F., Amakawa, L., \& Gould, M.S. (2013). Suicidal adolescents' experiences with bullying perpetration and victimization during high school as risk factors for later depression and suicidality. Journal of Adolescent Health, 53(1), 37-42. doi: 10.1016/j.jadohealth.2012.12.008

Leader, H., Singh, J., Ghaffar, A., \& de Silva, C. (2018). Association between bullying and pediatric psychiatric hospitalizations. SAGE Open Medicine, 6, 1-7. doi: $10.1177 / 2050312117750808$

Liu, Y, \& Lu, Z. (2012). Chinese high school students' academic stress and depressive symptoms: gender and school climate as moderators. Stress and Health, 28(4), 340-346. doi: 10.1002/smi. 2418

Landstedt, E., \& Gadin, K. G. (2012). Seventeen and stressed - Do gender and class matter? Health Sociology Review, 21(1), 82-89. doi:10.5172/hesr.2012.21.1.82

Lima, M. S. (2013). Correlatos valorativos do bullying: um estudo com estudantes e pais (Tese de doutorado). Universidade Federal da Paraíba, João Pessoa, Brasil. http://tede.biblioteca.ufpb.br/handle/tede/6939

Machado, W. L., \& Bandeira, D. R. Adaptação e validação da Depression, Anxiety and Stress Scale (DASS-21) para o português brasileiro. Manuscrito submetido à publicação.

Machado, S. F., Veiga, H. M. da S., \& Alves, S. H. de S. (2011). Níveis de estresse em alunos de $3^{\mathrm{a}}$ série do ensino médio. 
Universitas: Ciências da Saúde, 9(2), 35-52. doi: 10.5102/ UCS.V9I2.1362

Malecki, C. K., Demaray, M. K., Coyle, S., Geosling, R., Rueger, S. Y., \& Becker L. D. (2015). Frequency, power differential, and intentionality and the relationship to anxiety, depression, and self-esteem for victims of bullying. Child \& Youth Care Forum, 44(1), 115-131. doi:10.1007/ s10566-014-9273-y

Malta, D. C., Prado, R. R., Dias, A. J. R.F., Mello, C. M., Silva, M. A. I., Costa, M. R., \& Caiaffa, W. T. (2014). Bullying e fatores associados em adolescentes brasileiros: análise da Pesquisa Nacional de Saúde do Escolar (PeNSE 2012). Revista Brasileira de Epidemiologia, 17(1), 131-154. doi: 10.1590/1809-4503201400050011

Malta, D. C., Mello, F. C. M. de, Prado, R. R. do, Sá, A. C. M. G. N. de, Marinho, F., Pinto, I. V., Silva, M. M. A. da, \& Silva, M. A. I. (2019). Prevalência de bullying e fatores associados em escolares brasileiros, 2015. Ciência \& Saúde Coletiva, 24(4), 1359-1368. doi:10.1590/1413-81232018244.15492017

Marcolino, E. de C., Cavalcanti, A. L., Padilha, W. W. N., Miranda, F. A. N de, \& Clementino, F. de S. (2018). Bullying: prevalência e fatores associados à vitimização e à agressão no cotidiano escolar. Texto \& Contexto - Enfermagem, 27(1), e5500016. 2018.doi: 10.1590/0104-07072018005500016

Marques, C. P., Gasparotto, G. S., \& Coelho, R. W. (2015). Fatores relacionados ao nível de estresse em adolescentes estudantes: uma revisão sistemática. Salusvita, 34(1), 99108. https://secure.usc.br/static/biblioteca/salusvita/salusvita_v34_n1_2015_art_07.pdf

Martins, M. J. D. (2009). Maus-tratos entre adolescentes na escola. Lisboa, Portugal: Editorial.

Melim, M., \& Pereira, B. (2013). Bullying, gênero e idade. Em P. Silva, S. Souza, \& I. Neto (eds.), O desenvolvimento humano: perspectivas para o século XXI-Memória, lazer e atuação profissional (vol. 1, pp. 292-316). São Luís, MA: EDUFMA.

Menegotto, L. M. O., Pasini, A., \& Levandowski, I. G. (2013). O bullying escolar no Brasil: uma revisão de artigos científicos. Revista Psicologia: Teoria e Prática, 15(2), 203-215. http://pepsic.bvsalud.org/pdf/ptp/v15n2/16.pdf

Moore, S. E., Norman, R. E., Suetani, S., Thomas, H. J., Sly, P. D., \& Scott, J. G. (2017). Consequences of bullying victimization in childhood and adolescence: A systematic review and meta-analysis. World Journal of Psychiatry, 7(1), 6076. doi: 10.5498/wjp.v7.i1.60

Moraes, B. C. de, \& Hutz, C. S. (2012). Bullying: prevalência, implicações e diferenças entre os gêneros. Psicologia Escolar e Educacional, 16(1), 35-44. http://www.redalyc. org $/ \mathrm{html} / 2823 / 282323570004 /$

Moreira, L. C. O., \& Bastos, P. R. H. O. (2015). Prevalência e fatores associados à ideação suicida na adolescência: revisão de literatura. Psicologia Escolar e Educacional, 19(3), 445-453. doi: 10.1590/2175-3539/2015/0193857.
Moreno, E. A. C., Silva, A. P. da, Ferreira, G. A., Silva, F. P. da, Frazão, I. da S., \& Cavalcanti, A. M. T. de S. (2012). Perfil epidemiológico de adolescentes vítimas de bullying em escolas públicas e privadas. Revista Enfermagem UERJ, 20(6), 808-813. https://www.e-publicacoes.uerj. br/index.php/enfermagemuerj/article/view/6030/4337

Oliveira, W. A., Silva, J. L., Yoshinaga, A. C. M., \& Silva, M. A. I. (2015). Interfaces entre família e bullying escolar: uma revisão sistemática. Psico-USF, 20(1), 121-132. doi: 10.1590/1413-82712015200111

Pabian, S., \& Vandebosch, H. (2016). An investigation of short-term longitudinal associations between social anxiety and victimization and perpetration of traditional bullying and cyberbullying. Journal of Youth and Adolescence, 45(2), 328-339. doi: 10.1007/s10964-015-0259-3

Paixão, G., Santos, N., Matos, L., Santos, C., Nascimento, D., Bittencourt, I., \& Silva, R. (2014). Violência escolar: percepções de adolescentes. Revista Cuidarte, 5(2), 717-22. doi:10.15649/cuidarte.v5i2.83

Patias, N. D., Machado, W. L., Bandeira, D. R., \& Dell'Aglio, D. D. (2016). Depression, Anxiety and Stress Scale (DASS-21) - Short Form: adaptação e validação para adolescentes brasileiros. Psico-USF, 21(3),459-469. doi: 10.1590/1413-82712016210302

Romo, M. L., \& Kelvin, E. A. (2016). Impact of bullying victimization on suicide and negative health behaviors among adolescents in Latin America. Revista Panamericana de Salud Pública, 40(5), 347-355. https://www.scielosp.org/scielo.php?script $=$ sci_arttext\&pid $=$ S102049892016001100347\&lng = en\&nrm = iso\&tlng $=$ en

Sandoval Ato, R., Vilela Estrada, M. A., Mejia, C. R., \& Caballero Alvarado, J. (2018). Suicide risk associated with bullying and depression in high school. Revista Chilena de Pediatría, 89(2), 208-215. doi:10.4067/ s0370-41062018000100209

Santos, J. O., \& Santos, R. M. D. S. dos (2013). Bullying: o novo fenômeno da violência escolar. Revista Brasileira de Educação e Saúde, 1(1), 15-23. http://www.gvaa.com.br/ revista/index.php/REBES/article/view/1957

Santos, M. M., Perkoski, I. R., \& Kienen, N. (2015). Bullying: atitudes, consequências e medidas preventivas na percepção de professores e alunos do ensino fundamental. Temas em Psicologia, 23(4), 1017-1033. doi:10.9788/TP2015.4-16

Santrock, J. W. (2014). Adolescência. Porto Alegre, RS: Artmed.

Schermann, L. B., Béria, J. U., Jacob, M. H. V. M., Arossi, G., Benchaya, M. C., Bisch, N. K., \& Rieth, S. (2014). Estresse em adolescentes: estudo com escolares de uma cidade do sul do Brasil. Aletheia, (43-44), 160-173. http://pepsic. bvsalud.org/scielo.php?script=sci_arttext\&pid=S141303942014000100012\&lng $=$ pt\&tlng $=$ pt.

Segundo, P. R. R. E., Souza, A. F. G. de, Souza, D. K. de, Oliveira, H. G. de, Bueno, F. F., \& Prandi, L. R. (2016). Bullying escolar: os danos sociais e os aspectos jurídicos do 
fenômeno. Educere-Revista da Educação da UNIPAR, 15(2), 219-237. doi: 10.25110/educere.v15i2.2015.5621

Seixas, S. R. (2009). Diferenças de género nos comportamentos de bullying: contributos da neurobiologia. Interaçções, 5(13), 63-97. doi:10.25755/int.398

Silva, C. S., \& Costa, B. L. D. (2016). Opressão nas escolas: o bullying entre estudantes do ensino básico. Cadernos de Pesquisa, 46(161), 638-663. doi:10.1590/198053143888

Silva, E. F. J., \& Cabral, R. V. (2015, 20-22 out.). Como combater o bullying na escola e na sociedade. Resumos do Congresso Internacional de educação e inclusão, 1 (p. 1). Campina Grande: Editora Realize.

Simões, S., Ferreira, J. J., Braga, S., \& Vicente, H. T. (2015). Bullying, vinculação e estilos educativos parentais em adolescentes do $3^{\circ}$ ciclo do ensino básico. Revista Portuguesa de Investigação Comportamental e Social, 1(1), 30-41. http://rpics.ismt.pt/index.php/ISMT/issue/view/3/showToc

Sino, R., Arnold, E., Dourada, S., Langdon, S., Anderson, U., \& Bryant, U. (2014). Percepções e correlatos psicossociais do bullying entre os jovens indianos de Lumbee. American Indian and Alaska Native Mental Health Research, 21(1), 1-17. doi: 10.5820/aian.2101.2014.1.

Soares, A. K. S., Gouveia, V. V., Gouveia, R. S. V., Fonsêca, P. N. da, \& Pimentel, C. E. (2015). Escala Califórnia de Vitimização do Bullying (ECVB): evidências de validade e consistência interna. Temas em Psicologia, 23(2), 481-491. doi:10.9788/TP2015.2-18

Sousa, M. M. M. de, \& Stelko-Pereira, A. C. (2016). Relações entre violência escolar, gênero e estresse em pré-adolescentes. Revista Eletrônica de Educação, 10(1), 110-127. doi:10.14244/198271991304
Sousa, G. S. de, Santos, M. S. P. dos, Silva, A. T. P., Perrelli, J. G. A. da, \& Sougey, E. B. (2017). Suicide in childhood: a literatura review. Ciência \& Saúde Coletiva, 22(9), 3099-3110. doi:10.1590/1413-81232017229.14582017

Sprinthall, N. A., \& Collins, W. A. (2008). Psicologia do adolescente: uma abordagem desenvolvimentista ( $4^{\mathrm{a}}$ ed.). Lisboa, Portugal: Fundação Calouste Gulbenkian.

Stapinski, L. A., Araya, R., Heron, J., Montgomery, A. A, \& Stallard, P. (2015) Peer victimization during adolescence: concurrent and prospective impact on symptoms of depression and anxiety. Anxiety, Stress \& Coping, 28(1), 105120. doi: 10.1080/10615806.2014.962023

Stelko-Pereira, A. C., \& Williams, L. (2012). Desenvolvimento de rede para enfrentar o bullying: lições canadenses. Psicologia Escolar e Educacional, 16(2), 349-351. doi: 10.1590/S1413-85572012000200019

Ulbrich, G. D. S., Oselame, G. B., de, \& Oliveira, E. M., Neves, E. B. (2017). Motivadores da ideação suicida e a autoagressão em adolescentes. Adolescência \& Saúde, 14(2), 4046. https://www.researchgate.net/publication/318489665/ download

Vieira, I. S., Torales, A. P. B., Vargas, M. M., \& Oliveira, C. C. da C. (2016). Atitudes de alunos expectadores de práticas de bullying na escola. Ciência, Cuidado e Saúde, 15(1), 163170. doi: 10.4025/cienccuidsaude.v15i1.29403

Waseem, M., \& Nickerson, A. (2017). Bullying. Treasure Island, FL: StatPearls Publishing.

Werlang, B. S. G., Borges, V. R., \& Fensterseifer, L. (2004). Estudo de fidedignidade e validade da Escala de Ideação Suicida de Beck. Em B. S. G. Werlang, \& N. J. Botega (eds.), Comportamento suicida (pp. 189-193). Porto Alegre, RS: Artmed. 